\title{
A report on the laboratory performance of the spectroscopic detector arrays for SPIRE/HSO
}

Hien T. Nguyen, James J. Bock, Peter Ringold, John Battle, Steven C. Elliott, et al.

Hien T. Nguyen, James J. Bock, Peter Ringold, John Battle, Steven C. Elliott, Anthony D. Turner, Mark Weilert, Viktor V. Hristov, Bernhard Schulz, Ken Ganga, L. Zhang, Jeffrey W. Beeman, Peter A. R. Ade, Peter C. Hargrave, "A report on the laboratory performance of the spectroscopic detector arrays for SPIRE/HSO," Proc. SPIE 5498, Millimeter and Submillimeter Detectors for Astronomy II, (8 October 2004); doi: 10.1117/12.548974

Event: SPIE Astronomical Telescopes + Instrumentation, 2004, Glasgow, United Kingdom 


\title{
A Report on Laboratory Performance of the Spectroscopic Detector Arrays for SPIRE/HSO
}

\author{
H.T. Nguyen, J.J. Bock, P. Ringold, J. Battle, S.C. Elliott, A.D. Turner, M. \\ Weilert \\ Jet Propulsion Laboratory \\ California Institute of Technology, Pasadena, CA 91109 USA \\ email: Hien.T.Nguyen@jpl.nasa.gov, James.Bock@jpl.nasa.gov, \\ Peter.Ringold@jpl.nasa.gov \\ John.O.Battle@jpl.nasa.gov,Steven.Elliott@jpl.nasa.gov, \\ Anthony.D.Turner@jpl.nasa.gov, \\ Mark.Weilert@jpl.nasa.gov \\ V. V. Hristov \\ California Institute of Technology, Mail Stop 59-33, Pasadena, CA 91125 USA \\ email:vvh@astro.caltech.edu \\ B. Schulz, K. Ganga, L. Zhang \\ IPAC \\ California Institute of Technology, Mail Stop 59-33, Pasadena, CA 91125 USA \\ email:bschulz@ipac.caltech.edu,@ipac.caltech.edu
}

J.W. Beeman

Berkeley National Laboratory, Berkeley, CA 94720 USA

email: jwbeeman@1bl.gov

P.A.R. Ade, P.C. Hargrave

University of Wales, Cardiff

p.hargrave@qmw.ac.uk

\begin{abstract}
We report the performance of the flight bolometer arrays for the Spectral and Photometric Imaging REceiver (SPIRE) instrument to be on board of the Herschel Space Observatory (HSO). We describe the test setup for the flight Bolometric Detector Assembly (BDA) that allows the characterization of its performance, both dark and optical, in one instrument's cool down. We summarize the laboratory procedure to measure the basic bolometer parameters, optical response time, optical efficiency of
\end{abstract}


bolometer and feedhorn, dark and optical noise, and the overall thermal conductance of the BDA unit. Finally, we present the test results obtained from the two flight units, Spectroscopic Long Wavelength (SLW) and Spectroscopic Short Wavelength (SSW).

\section{INTRODUCTION}

We refer the reader to Tuner et al. (2001) and references therein for extensive discussion on the subject of bolometry and the fabrication of silicon nitride micromesh bolometer array. In this section we introduce the basic notation that will be used in later discussion.

Bolometer Resistance is a function of the temperature, $T$, such that:

$$
R=R_{o} \exp (\sqrt{ } \Delta / T)
$$

And the thermal conductance,

$$
G=G_{o}(T / .3)^{\beta}
$$

$G_{o}$ is the thermal conductance $(\mathrm{W} / \mathrm{K})$ measured at $300 \mathrm{mK}$.

\section{BODAC - THE BOLOMETRIC DETECTOR ASSEMBLY TESTBED}

In this section we are going to give a brief description of the instrument. A more detail account of BoDAC with optics is expected to be presented elsewhere.

\section{The Cryostat}

BoDAC is a $45 \mathrm{~cm}$ by $107 \mathrm{~cm}$ liquid helium cryostat (built by Janis Research). It has a liquid nitrogen reservoir (25 liters) to cool down to $77 \mathrm{~K}$ and a helium reservoir (25 liters) to reach $4.2 \mathrm{~K}$. The final cold operating temperature is attained by a closed cycle ${ }^{4} \mathrm{He} /{ }^{3} \mathrm{He} /{ }^{3} \mathrm{He}$ fridge (built by Chase Research). The fridge has an intermediate stage at $360 \mathrm{mK}$ to intercept $\sim 30 \mu \mathrm{W}$ parasitic load from $4.2 \mathrm{~K}$ bath; the thermal load is dominated by electrical wires for 144 detectors. The fridge cycle doesn't require pumping on liquid helium bath and can be automated to cool the BDA to the operating temperature at $250 \mathrm{mK}$ for more than 24 hours.

\section{The Test Setup}

The BDA mount, also known as the intercooler flange or ICF, is connected to the 360 $\mathrm{mK}$ intercooler stage (IC) and is supported by four vespel legs to provide thermal isolation to $4.2 \mathrm{~K}$ Helium bath. By design, the bolometer is thermally connected to the ${ }^{3} \mathrm{He} /{ }^{4} \mathrm{He}$ fridge (called the ultracold or UC stage at $250 \mathrm{mK}$ ) by means of a copper coldfinger firmly attached to the feedhorn plate. A cold calibrator is mounted at about 2 $\mathrm{cm}$ in front of the BDA, completely overfill the beam of each feedhorn. An array of pin holes is drilled into the cold calibrator in order to allow the detectors to look out to the room. In normal condition, the pinhole allows less than $0.05 \mathrm{pW}$ of thermal emission 
from the window. The filter on the BDA itself is broad band, and in order to provide single-mode bandwidth, we mount a custom made edge filter in front of the cold calibrator. Note that there is no optics except the feedhorn, and the cold calibrator setup ensures zero high frequency leakage.

There are a heater and a thermometer at each of the three stages of temperature, UC, IC, and ICF. These are for measuring the BDA thermal conductance.
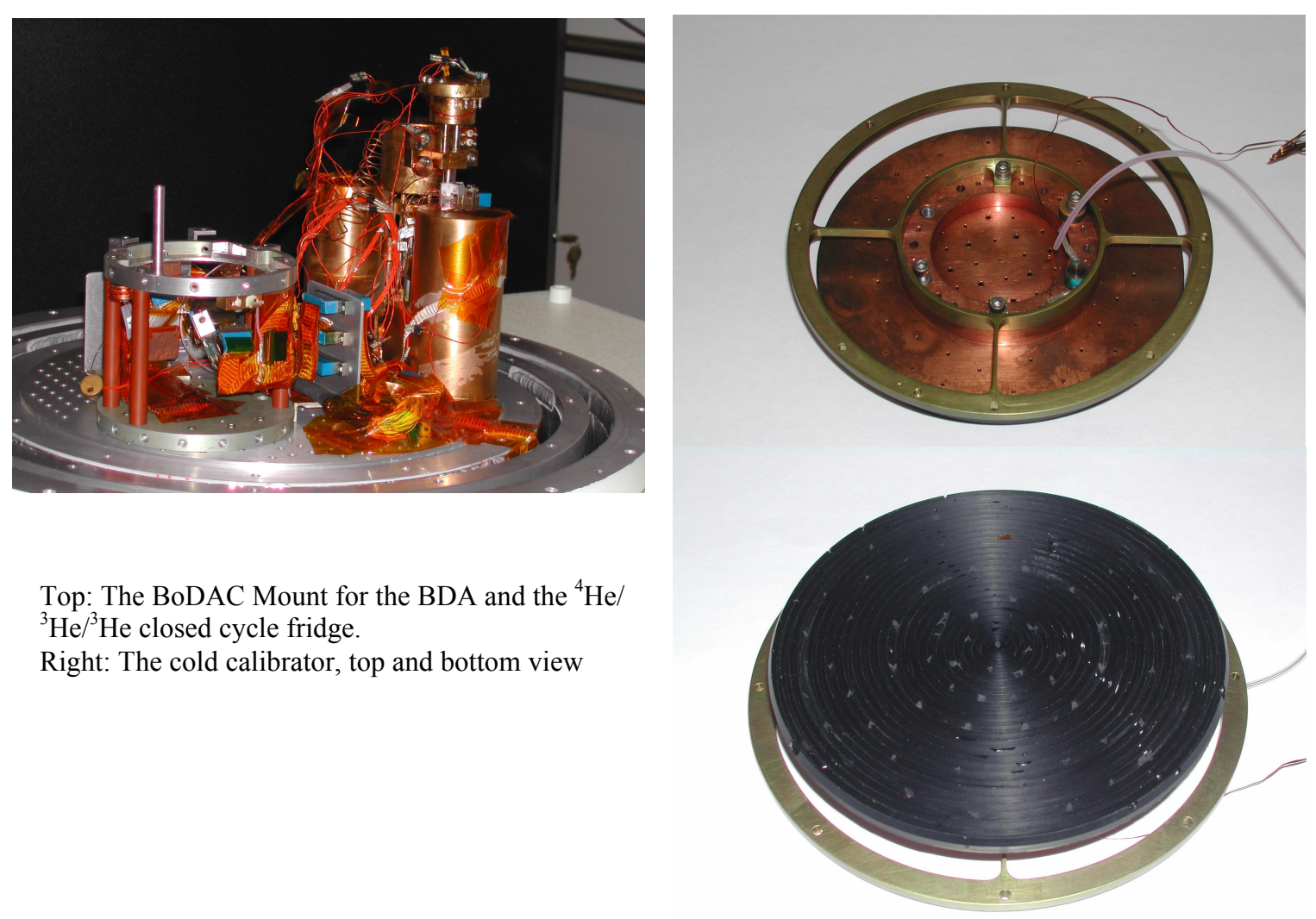

Top: The BoDAC Mount for the BDA and the ${ }^{4} \mathrm{He} /$ ${ }^{3} \mathrm{He} /{ }^{3} \mathrm{He}$ closed cycle fridge.

Right: The cold calibrator, top and bottom view

\section{The Electronics}

Each of the differential bolometer signal is buffered by a pair of low noise U401 junction field effect transistor. The complete JFET resides at the liquid-nitrogen stage, with a total of six modules each has 24 JFET pairs, and is maintained at $135 \mathrm{~K}$ by its own electrical power. The JFET differential output is amplified using INA103 located outside the cryostat and demodulated by a lock-in amplifier circuit before digitized and stored in the computer. The readout electronics and data acquisition system are capable of acquiring 144 channels simultaneously. Our software allows immediate diagnostic and data analysis.

\section{LABORATORY TEST PROCEDURE}

In this section we give a summary of the test procedures for the measurement of, i. the bolometer parameters, ii. the bolometer time constant, iii. the bolometer and feedhorn optical efficiency, iv. the bolometer dark noise, v. the BDA thermal conductance and vi. the BDA thermal time constant. 
1. Measure the Bolometer Parameters: $R_{o}, \Delta, G_{o}$ and $\beta$

We pump on the liquid Helium bath in order to reduce the temperature of the cold calibrator to less than $1.5 \mathrm{~K}$ and effectively achieve the "dark" condition for the load curve measurements. The pumping also further reduces thermal loading via the detector kapton cable on the BDA. The true bolometer temperature is calibrated with commercial Germanium Resistance Thermometer (Lakeshore) located at the top of the cold finger attached to the feedhorn array. (Note that the "hot" end of the kapton cable is often less than $.5 \mathrm{~K}$ due to contact with the $360 \mathrm{mK}$ stage).

Load curve is obtained at each temperature setting (stepping the bolometer temperature from 280 to $750 \mathrm{mK}$ ). We vary the bias voltage and record the bolometer voltage.

Since,

$$
V=V_{\text {bias }} R /\left(R+R_{\text {load }}\right)
$$

The resistance of the bolometer at the bath temperature, ie., near the zero electrical power, can be immediately obtained:

$$
R=R_{\text {load }} V /\left(V_{\text {bias }}-V\right)
$$

$R_{o}$ and $\Delta$ can be simply obtained using (1).

The current at each bias point is,

$$
I=V / R
$$

The electrical power, $P$, at each bias point is then

$$
P=V^{2} / R
$$

At equilibrium,

$$
P=\int G d T=G_{o} /(1+\beta)(.3)^{-\beta}\left[T^{\beta+1}-T_{o}{ }^{\beta+1}\right],
$$

where $T$ is the intrinsic bolometer temperature, which may be deduced from (1), and $T_{o}$ is the bath temperature. $\mathrm{G}_{\mathrm{o}}$ and $\beta$ may be readily extracted using (5) and (6) (albeit a nonlinear fit).

\section{Measure the Bolometer Time Constant}

As it has been described in the Setup section, the BDA is looking out to the room thru the cold calibrator's pinhole (these pinholes allow less than $0.05 \mathrm{pW}$ of thermal emission from being capped off with aluminum window at room temperature). We illuminate the detectors with a modulated thermal source $(\mathrm{T}>800 \mathrm{C})$ and record the bolometer voltage, 
$V$, at fixed DC bias. The measurement is done for frequency range from 0 to $150 \mathrm{~Hz}$ (the detector sampling frequency is $300 \mathrm{~Hz}$ ). We repeat these measurements for the three fixed DC biases at 15,25 and $40 \mathrm{mV}$.

The time constant, $\tau$, is defined to be:

$$
V(f)=V(f=0) / \sqrt{ }\left(1+4 \pi^{2} f^{2} \tau^{2}\right)
$$

\section{Measure the Optical Efficiency, $h$}

The cold calibrator temperature is controlled by a heater and is weakly thermally linked to $4.2 \mathrm{~K}$ bath. The incident power, $Q$, can be simply estimated (see, for example, Richards (1993)), corrected for the transmission of the filters and waveguide cutoff, $\tau(x)$,

$$
Q=2 k_{B}^{2} / h T_{B B}^{2} \int \tau(x) x /\left(e^{x}-1\right) d x
$$

where $T_{B B}$ is the temperature of the cold calibrator, and $x=h v / k_{B} T_{B B}$. The integral is done from 0 to $\infty$. Note that we assume single mode throughput, ie., $A \Omega=\lambda^{2}$. We record the load curve under different optical loads. The difference in absorbed power of each detector is determined by measuring the difference of electrical power at a fixed resistance (see, for example, Turner et al. (2001)). The optical efficiency, $h$, is

$$
\mathrm{h}=\text { absorbed power / incident power }
$$

At high enough temperature, radiation from the cold calibrator may heat up the base temperature, which can be read off thermistor channel. For a given change in the base temperature, $\Delta T$, correction for absorbed power of each detector then may be apply, such that

$$
\Delta P=G_{o} \Delta T
$$

where $G_{o}$ is the thermal conductance of the bolometer.

\section{Measure the Dark and Optical Noise}

Bolometers are ac-biased at $150 \mathrm{~Hz}$. The differential signal is then amplified and demodulated using an analog lock-in amplifier (LIA). The total electronics noise (bias+JFET+preamp+LIA) is about $10 \mathrm{nV} / \mathrm{rt}(\mathrm{Hz})$. The total "dark noise" is measured when the cold calibrator is at $4.2 \mathrm{~K}$, and the total optical noise when the cold calibrator is at higher temperature. We also record the DC loadcurve for each optical loading in order to determine directly the responsivity of the detector,

$$
S_{d c}=1 / 2 I(Z / R-1) /\left(Z / R_{\text {load }}+1\right)
$$

So that, 


$$
N E P_{\text {total }}=N E V_{\text {total }} / S_{d c}
$$

Where, $N E V$ is the measured noise equivalent voltage.

We note that the total electronic gain is determined by multiplying the preamp gain $g_{\text {preamp }}$ and the lock-in amplifier gain $g_{\text {LIA }}$. We find the total gain obtained this way is consistent with the value obtained by measuring the expected total voltage noise of a fixed resistor (it may help to choose a high enough resistance at room temperature so that the contribution from the amplifier noise is negligible). In particular, the differential johnson noise output from a resistor $R_{\text {simulated }}$ with load resistors $R_{\text {Load }}$ amplified by $g_{\text {preamp }}$ and demodulated by a lock-in amplifier with gain $g_{L I A}$ is,

$$
N E P_{\text {measured }}=\sqrt{ } 2 \times g_{\text {preamp }} \times g_{L I A} \times \sqrt{ }\left(4 k_{B} T R_{\text {eff }}\right)
$$

where

$$
1 / R_{\text {eff }}=1 / R_{\text {simulated }}+1 / R_{\text {Load }}
$$

The $D Q E$ is defined to be,

$$
D Q E=\left(N E P_{\text {photon }} / N E P_{\text {total }}\right)^{2}
$$

where,

$$
\left(N E P_{\text {photon }}\right)^{2}=4 k_{B}{ }^{3} / h T_{B B}{ }^{3} \int \tau(x)\left[x^{2} /\left(e^{x}-1\right)+x^{2} /\left(e^{x}-1\right)^{2}\right] d x
$$

Note that the first term is the shot noise, and the second the bose-einstein. Again, we assume single mode throughput and the integral is done from 0 to $\infty$.

The measured value of $D Q E$ can be compared with the predicted $D Q E_{c a l}$, which is now described below.

Let's again define the physical setup. A bolometer at temperature $T$ has a resistance $R$, as defined in (1). The bolometer is connected to a bath temperature $T_{o}$ via a thermal link with thermal conductance $G$, as defined in (2). At some bias current $I$, the bolometer has an impedance $Z$ such that,

$$
\begin{aligned}
& Z / R=(I / V)(d V / d I)=[-1-G T /(P \alpha)] /[1-G T /(P \alpha)] \\
& \text { and } \quad \alpha=(T / R)(d R / d T)=-0.5(\Delta / T)^{1 / 2}
\end{aligned}
$$

We compute the noise components as the following,

$$
\begin{aligned}
& N E P_{\text {johnson }}{ }^{2}=\left(4 k T^{3} G^{2}\right) /\left(P \alpha^{2}\right) \\
& N E P_{\text {phonon }}{ }^{2}=\left[\left(4 k T_{o}^{2} G\right)(\beta+1)\left(\left(T / T_{o}\right)^{2 \beta+3}-1\right)\right] /\left[(2 \beta+3)\left(T / T_{o}\right)^{\beta}\left(\left(T / T_{o}\right)^{\beta+1}-1\right)\right]
\end{aligned}
$$




$$
\begin{aligned}
& N E P_{\text {Rload }}^{2}=\left[4 k T_{o} / 2 R_{L}\right]|2 Z I /[(Z / R)-1]|^{2} \\
& N E P_{\text {amp }}{ }^{2}=\left(V_{n} / S_{d c}\right)^{2}
\end{aligned}
$$

All the different noise contributions are un-correlated and so the detector noise is defined to be,

$$
N E P_{\text {detector }}^{2}=2 N E P_{\text {johnson }}^{2}+N E P_{\text {phonon }}^{2}+2 N E P_{\text {Rload }}^{2}+2 N E P_{\text {amp }}^{2}
$$

The total calculated noise is then,

$$
\begin{aligned}
N E P_{\text {cal }}{ }^{2} & =N E P_{\text {photon }}{ }^{2}+N E P_{\text {detector }}^{2} \\
\text { and } \quad D Q E_{\text {cal }} & =N E P_{\text {photon }}{ }^{2} /\left(N E P_{\text {photon }}{ }^{2}+N E P_{\text {detector }}{ }^{2}\right)
\end{aligned}
$$

\section{Measure the BDA Thermal Conductance}

As described in the Setup, there are 3 temperature stages here,

$\mathrm{UC}=$ Ultra Cold stage, $250 \mathrm{mK}$, strapped to the BDA

$\mathrm{IC}=$ Inter Cooler stage, about $360 \mathrm{mK}$, strapped to BDA "1.1 K" flange

$\mathrm{ICF}=\mathrm{IC}$ Flange, basically the mounting point of the BDA

The ICF is connected to the IC through a weak thermal link so we can heat up the ICF to $2 \mathrm{~K}$. The basic steps are:

1. Calibrate the UC stage $\mathrm{T}_{\mathrm{uc}}$ vs. $\mathrm{P}_{\mathrm{uc}}$ by applying heat directly to the UC

2. Calibrate the conductivity internal to the fridge by heating up the IC stage.

3. Heat up the ICF and watch $T_{\text {uc }}$ vs. $T_{\text {icf. }}$ This allows us to calculate $\operatorname{Puc}\left(T_{u c}, T_{i c}\right)$ vs. $\mathrm{T}_{\text {icf. }}$

4. Fit $P_{u c}$ vs. $T_{\text {icf }}$

\section{Measure the BDA Thermal Time Constant}

We determine the time constant of the BDA by

1. Set the fridge on a fixed temperature, $250 \mathrm{mK}$.

2. Set the bolometer bias at a fixed dc voltage, nominally $15 \mathrm{mV}$

3. Start continuous recording of bolometer voltage for $30 \mathrm{~min}$

4. Apply power on the fridge to bring it to a higher temperature

The BDA time constant is determined by measuring the time the bolometer to come to equilibrium.

\section{THE S/LW AND S/SW BDA'S}


The S/LW and S/SW BDA are the two spectroscopic arrays for SPIRE. The S/LW unit has 19 detectors and covers the waveband of 300 to $670 \mu \mathrm{m}$, and the S/SW has 37 detectors and covers the waveband of 200 to $300 \mu \mathrm{m}$. In addition, each array has two thermistors to provide temperature information in flight and a fixed resistor for diagnostic purpose (each array also has two additional "dark" channels, but the feedhorn array gap exposes them to light, thus making them no longer dark).

Figure 1 below shows the bolometer and the feedhorn array. The top panels, a and $\mathrm{b}$ are the bolometer and load resistor array, and the $300 \mathrm{mK}$ stage assembly of detector and feedhorn arrays for the S/SW. The bottom panels, $c$ and d, are for S/LW. Figure e show the complete BDA assembly with the $300 \mathrm{mK}$ flight filter integrated on top. The Kevlar thread provides thermal isolation between $300 \mathrm{mK}$ stage and the $1.7 \mathrm{~K}$ flange. The two complete units were undergoing rigorous environment (vibration and thermal cycle) before being installed into BoDAC for electrical and optical characterization.
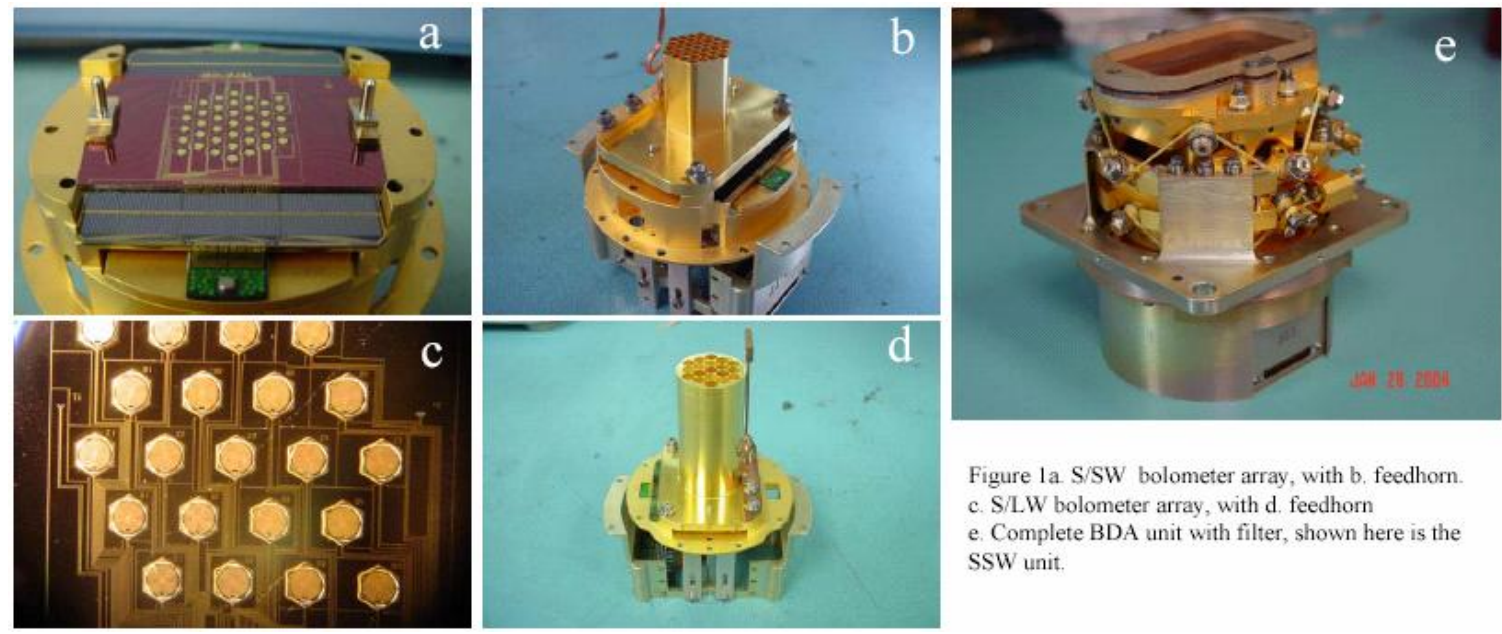

Figure 1a S/SW bolometer array, with $\mathrm{b}$, feedhorn. c. S/LW bolometer array, with d. feedhorn e. Complete BDA unit with filter, shown here is the SSW unit.

\section{THE PERFORMANCE DATA AND SUMMARY}

For the purpose of illustration, we show only plots for either the S/LW or S/SW unit, the median for both units is tabulated in table 1 and 2 .
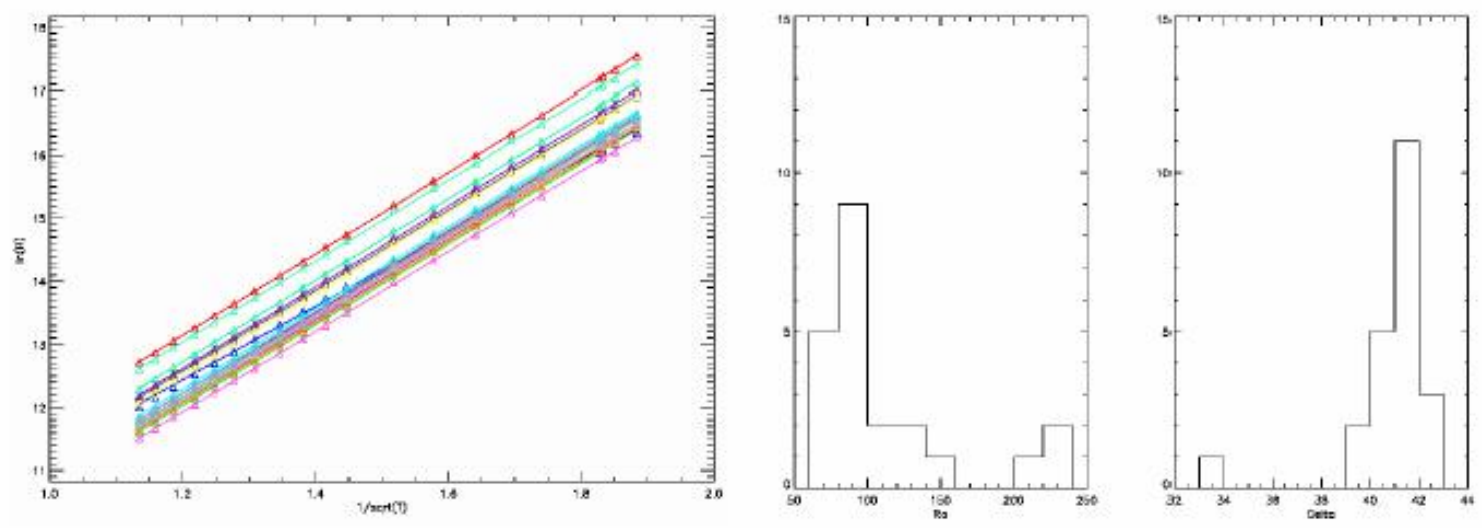

Figure 2. Left: $\ln (\mathrm{R})$ vs. $1 / \sqrt{ }$ T. Right: Histogram of $R_{o}$ and $\Delta$. 

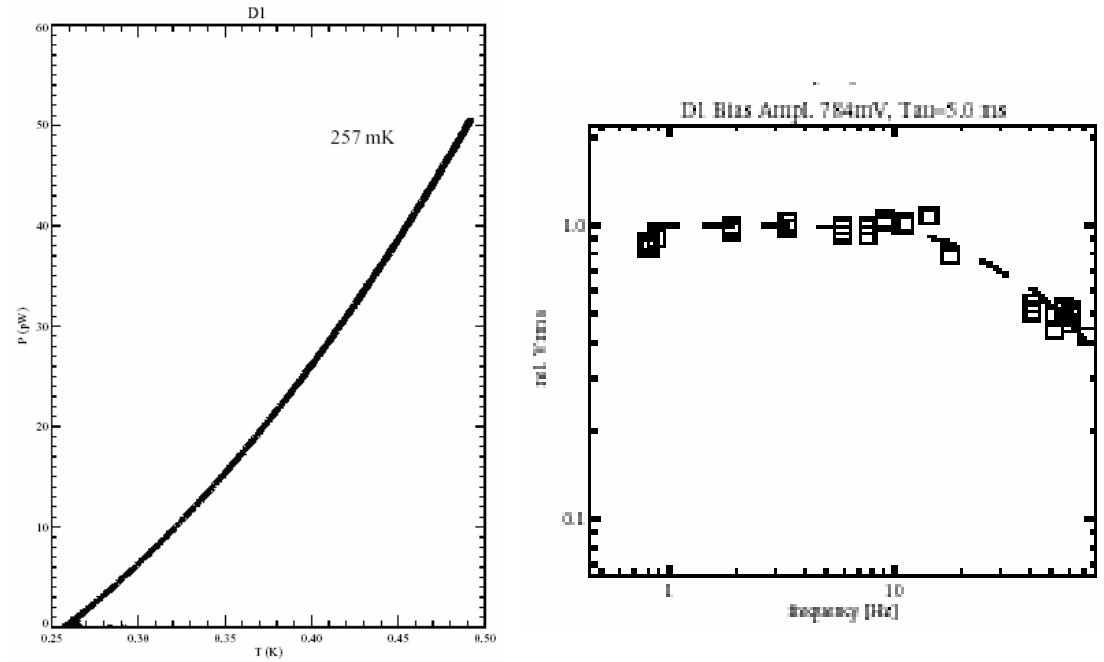

Figure 3. Left: Electrical Power, P, vs. Bolometer Temperature T. Right: Bolometer Response Time of the same pixel.
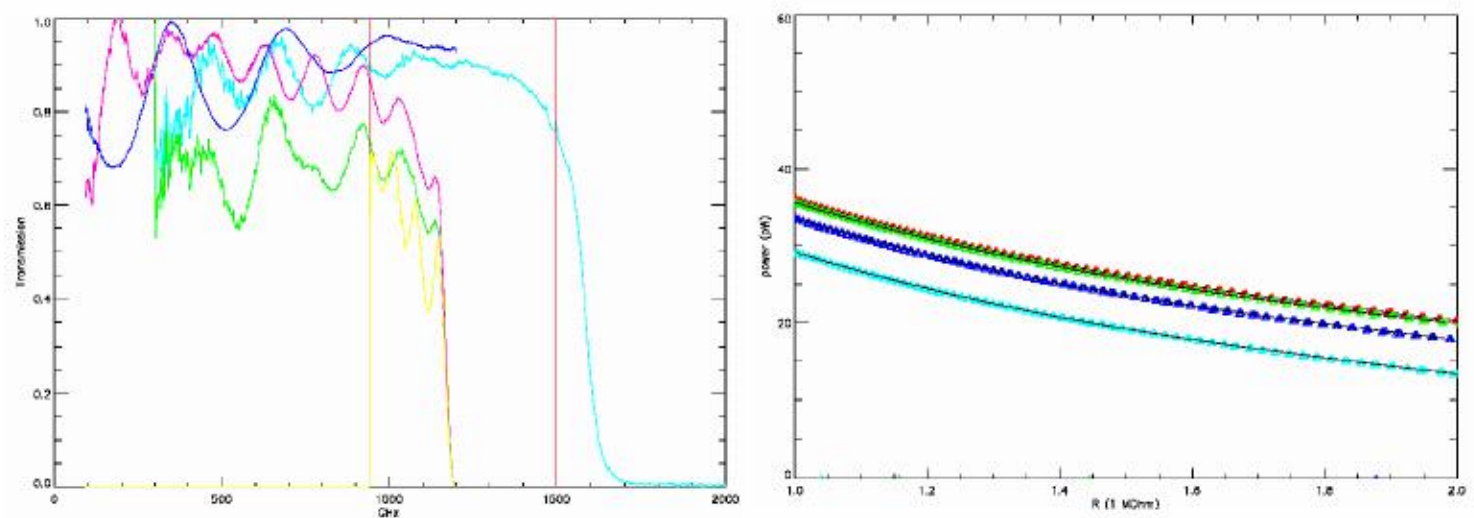

Figure 4. Left: The various filter and lens transmission and the result optical Bandpass (in yellow) of BoDAC S/SW unit. Right: A plot of power vs. R for four different optical loading for a typical pixel. 


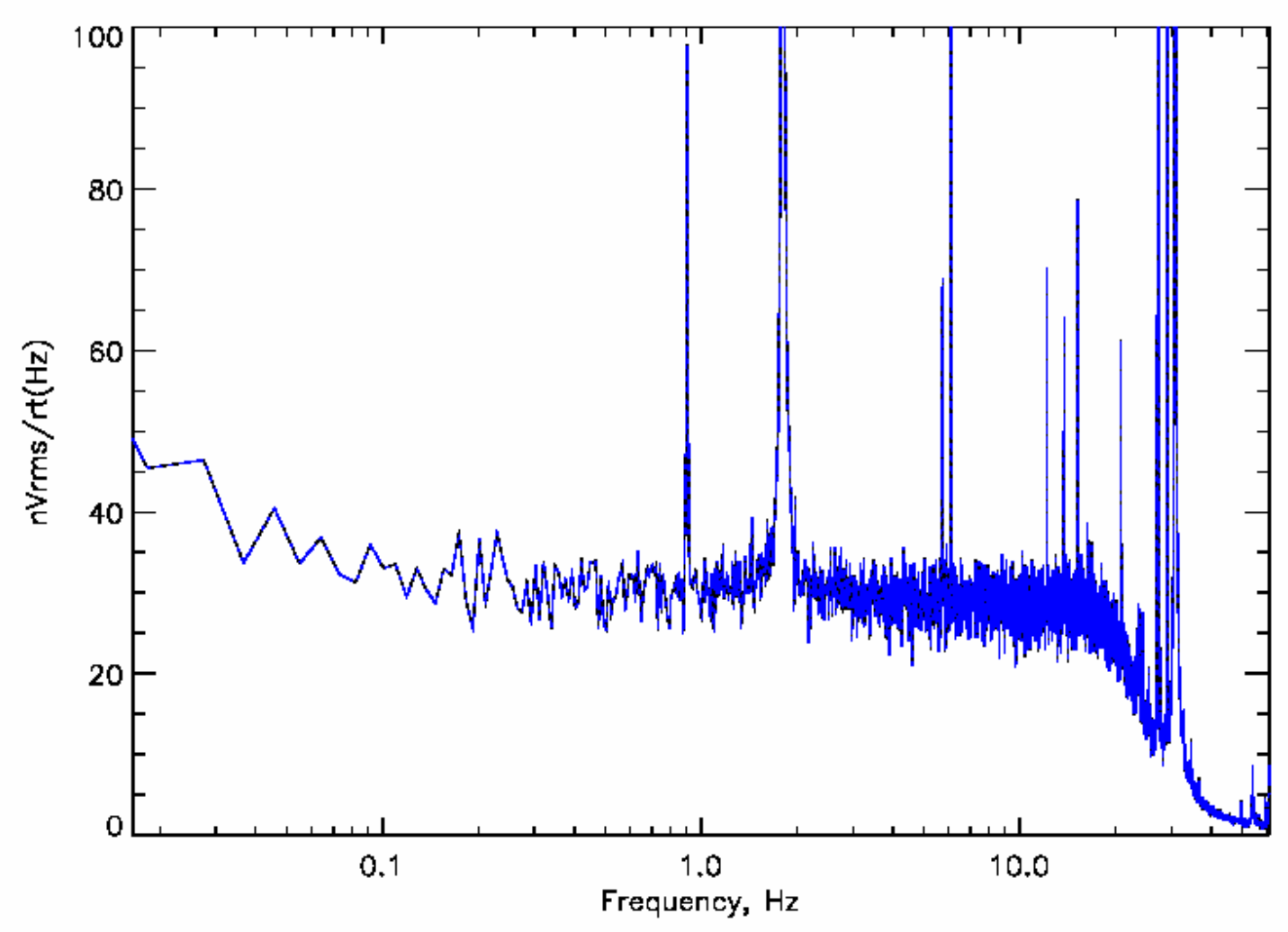

Figure 5. Noise spectra of a typical S/LW detector.

Above we presented some highlight of the performance data for the S/LW and S/SW BDA's. We are scheduled to conduct the same measurements for the three flight photometric arrays. These are Long, Medium and Short wavelengths for centered at 250, 350 and $500 \mu \mathrm{m}$. We expect to complete the measurement and delivery by the end of this year, 2004.

Acknowledgement: Funding for this work was provided by the Planck and Herschel Project at the Jet Propulsion Laboratory. 
TABLE 1

PERFORMANCE VERIFICATION MATRIX - PFM SLW BDA - S/N 10209800-1-008

BDA Performance

\begin{tabular}{|c|c|c|c|c|c|c|}
\hline Item & D. Value & Min Perf & Measured Median & Unit & Reference & Note \\
\hline Number of bad optical pixels & 52 & 55 & 0 & & BDA-PER-01 & \\
\hline (NEP photon/NEPtotal) 2 (derived) & $>0.6$ & $>0.5$ & 0.53 & & BDA-PER-02 & at $40 \mathrm{mV}$ bias \\
\hline (NEPphoton/NEPtotal) 2 (measured) & $>0.6$ & $>0.5$ & 0.54 & & BDA-PER-02 & at $40 \mathrm{mV}$ bias \\
\hline Optical efficiency* ${ }^{*}$ & $>0.7$ & $>0.65$ & 0.75 & & BDA-PER-05 & \\
\hline Detector time constant & $<4.2$ & $<14$ & 5.1 & $\mathrm{~ms}$ & BDA-PER-07 & at $25 \mathrm{mV}$ bias \\
\hline$\sqrt{\max x^{* *+}}$ & $<17$ & & 12.8 & $\mathrm{mV}$ & BDA-DRCU-22 & \\
\hline Calibration uniformity* & $>0.99$ & $>0.99$ & N/M & & BDA-PER-08 & \\
\hline Cross-talk $(n-n)^{* *}$ & $<0.01$ & $<0.05$ & N/M & & BDA-PER-09 & \\
\hline Cross-talk (non n-n) $)^{* *}$ & $<0.001$ & $<0.001$ & $\mathrm{~N} / \mathrm{M}$ & & BDA-PER-09 & \\
\hline $1 / \mathrm{f}$ knee frequency & $<30$ & $\mathrm{k} 100$ & $<30$ & $\mathrm{mHz}$ & BDA-PER-10 & at $25 \mathrm{mV}$ bias \\
\hline Average conducted heat load from $1.7 \mathrm{~K}$ & $<1.6$ & $<3.0$ & $<2.9$ & UW & BDA-TEC-06 & \\
\hline \multicolumn{7}{|c|}{ BDA Design Values (at $300 \mathrm{mK}$ ) } \\
\hline Item & Target & & Measured Median & Unit & Reference & Note \\
\hline Ro & 180.0 & & 90.2 & Dhms & BDA-SSSD & \\
\hline Delta & 41.8 & & 41.3 & $\mathrm{~K}$ & BDA-SSSD & \\
\hline R300 & 24.0 & & 11.2 & MOhms & BDA-SSSD & \\
\hline $\mathrm{G} 300$ & 170.0 & & 160.6 & DW/K & BDA-SSSD & \\
\hline Beta & 1.50 & & 1.26 & & BDA-SSSD & \\
\hline $\mathrm{C} 300$ & 1.00 & & 0.88 & $\mathrm{DJ} / \mathrm{K}$ & BDA-SSSD & \\
\hline RIr & 10.0 & & 12.1 & MOhms & BDA-SSSD & room temp \\
\hline Dark Sdc & 3.8 & & 3.1 & $28 \mathrm{~V} M \mathrm{~N}$ & derived & at $25 \mathrm{mV}$ bias \\
\hline Dark NEP $(1 \mathrm{~Hz})$, incl $10 \mathrm{nV} / \mathrm{rtHz}$ amp. noise & 5.6 & & 5.9 & e-17 WirtHz & derived & at $25 \mathrm{mV}$ bias \\
\hline Dark NEP $(0.1 \mathrm{~Hz})$, incl $10 \mathrm{nV} / \mathrm{rtHz}$ amp. noise & 5.6 & & 6.9 & e-17 WirtHz & derived & at $25 \mathrm{mV}$ bias \\
\hline$V \max$ & 0.0114 & & 0.0103 & Vrms & BDA-SSSD & \\
\hline BDA temperature rise from $1.7 \mathrm{~K}$ & $\angle 10$ & & $<11.6$ & $\mathrm{mK}$ & BDA-HCO-1 & \\
\hline BDA thermal time constant & $>100$ & & 200 & & BDA-HCO-2 & \\
\hline
\end{tabular}

"assumes vlower $=1.02$ vcutoff

"not tested

"*Thermistor values are not included

thermistors saturate at $31 \& 69 \mathrm{mV}$ bias at $0.3 \mathrm{~K}$

TABLE 2

PERFORMANCE VERIFICATION MATRIX - PFM SSW BDA - S/N 10209800-5-009

BDA Performance

\begin{tabular}{|c|c|c|c|c|c|c|}
\hline Item & D. Value & Min Perf & Measured Median & Unit & Reference & Note \\
\hline Number of bad optical pixels & 54 & 59 & 2 & & BDA-PER-01 & \\
\hline (NEPphoton/NEPtotal) 2 (derived) & 0.71 & 0.59 & 0.62 & & BDA-PER-02 & at $30 \mathrm{mV}$ bias \\
\hline Optical efficiency $y^{*}$ & $>0.7$ & & 0.70 & & BDA-PER-05 & \\
\hline Detector time constant & $<4.2$ & $<8$ & 3.9 & $\mathrm{~ms}$ & BDA-PER-07 & at $28 \mathrm{mV}$ bias \\
\hline$V \max *+*$ & $<17^{*+*+}$ & & 14.8 & $\mathrm{mV}$ & BDA-DRCU-22 & \\
\hline Calibration uniformity** & $>0.99$ & $>0.99$ & $\mathrm{~N} / \mathrm{M}$ & & BDA-PER-08 & \\
\hline Cross-talk $(n-n)^{* *}$ & $<0.01$ & $<0.05$ & $\mathrm{~N} / \mathrm{M}$ & & BDA-PER-09 & \\
\hline 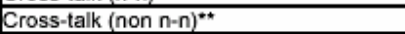 & 0.001 & 0.001 & $\mathrm{~N} / \mathrm{M}$ & & BDA-PER-09 & \\
\hline $1 / \mathrm{f} \mathrm{knee} \mathrm{frequency}$ & $<30$ & $<100$ & $<30$ & $\mathrm{mHz}$ & BDA-PER-10 & at $21.2 \mathrm{mV}$ bias \\
\hline Average conducted heat load from $1.7 \mathrm{~K}$ & 61.6 & $<3.0$ & $<3.5$ & $\overline{\mathrm{uW}}$ & BDA-TEC-06 & \\
\hline
\end{tabular}

BDA Design Values (at $300 \mathrm{mK}$ )

\begin{tabular}{|c|c|c|c|c|c|}
\hline Item & Target & Measured Median & Unit & Reference & Note \\
\hline RO & 180.0 & 93.1 & Ohms & BDA-SSSD & \\
\hline Delta & 41.8 & 42.0 & K & BDA-SSSD & \\
\hline $\mathrm{R} 300$ & 24.0 & 10.5 & MOhms & BDA-SSSD & \\
\hline G300 & 170.0 & 196.0 & $\mathrm{pW} / \mathrm{K}$ & BDA-SSSD & \\
\hline Beta & 1.50 & 1.29 & & BDA-SSSD & \\
\hline $\mathrm{C300}$ & 1.00 & 0.90 & $\mathrm{pJ} / \mathrm{K}$ & BDA-SSSD & \\
\hline RIr & 10.0 & 8.4 & MOhms & BDA-SSSD & oom temp \\
\hline Dark Sdc & 4.1 & 2.9 & $88 \mathrm{~V} / \mathrm{W}$ & BDA-SSSD & at $21.2 \mathrm{mV}$ bias \\
\hline Dark NEP $(1 \mathrm{~Hz})$, incl $10 \mathrm{nV} / \mathrm{rttHz}$ amp. noise & 5.6 & 6.9 & e-17 W/rthz & derived & at $21.2 \mathrm{mV}$ bias \\
\hline Dark NEP $(0.1 \mathrm{~Hz})$, incl $10 \mathrm{nV} / \mathrm{itHz}$ amp. noise & 5.6 & 7.5 & e-17 W/ritHz & derived & at $21.2 \mathrm{mV}$ bias \\
\hline Vmax & 10.3 & 11.6 & $\mathrm{mV} r \mathrm{rms}$ & BDA-SSSD & SSSD value in error \\
\hline BDA temperature rise from $1.7 \mathrm{~K}$ & $<10$ & $\leq 7.2$ & $\mathrm{mK}$ & BDA-HCO-1 & \\
\hline BDA thermal time constant & $\sim 100$ & 97 & $s$ & BDA-HCO-2 & \\
\hline
\end{tabular}

"assumes vlower $=1.02$ vcutoff

"not tested

"*Thermistor values are not included 


\section{References}

1. P.L. Richards, "Bolometers for infrared and millimeter waves," J. Appl. Phys. 76, 124 (1994). 